Trojanowska Paulina, Trojanowska Alina, Szydełko Joanna, Tywanek Ewa, Luczyk Robert Jan. The impaired response of circulating asprosin concentrations to glucose levels fluctuation may be one of the causes of type 2 diabetes - a narrative review. Journal of Education, Health and Sport. 2020;10(9):846-854. eISSN 2391-8306. DOI http://dx.doi.org/10.12775/JEHS.2020.10.09.102 https://apcz.umk.pl/czasopisma/index.php/JEHS/article/view/JEHS.2020.10.09.102 https://zenodo.org/record/4054287

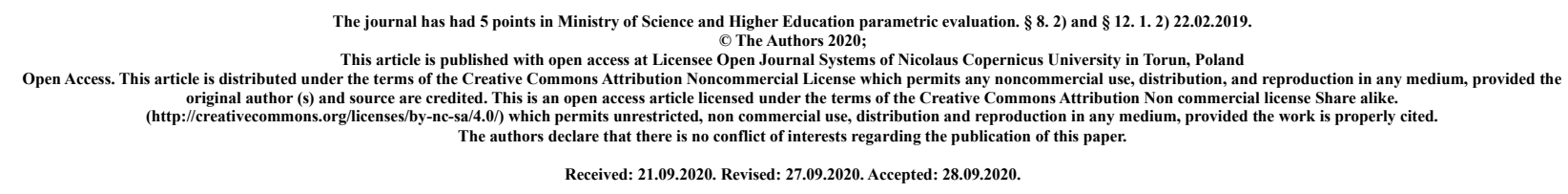

The impaired response of circulating asprosin concentrations to glucose levels fluctuation may be one of the causes of type 2 diabetes - a narrative review

\title{
Paulina Trojanowska ${ }^{1}$, Alina Trojanowska ${ }^{2}$, Joanna Szydełko $^{3}$, Ewa Tywanek ${ }^{4}$, Robert Jan Luczyk ${ }^{4}$
}

${ }^{1}$ Department of Pediatric Neurology and Department of Endocrinology, Medical University of Lublin, Poland

${ }^{2}$ Chair and Department of Pediatric Nursing, Faculty of Health Sciences, Medical University of Lublin

${ }^{3}$ Department of Endocrinology, Medical University of Lublin, Poland

${ }^{4}$ Chair of Internal Medicine and Department of Internal Medicine in Nursing, Medical University of Lublin, Poland

\author{
Authors \\ 1. Paulina Trojanowska, trojanowska_paulina@wp.pl,https://orcid.org/:0000-0002-5289-6473, \\ 2. Alina Trojanowska, trojanowska-a@wp.pl, https://orcid.org/0000-0002-4021-7509, \\ 3. Joanna Szydełko, jszydelko@interia.pl, https://orcid.org/0000-0003-3744-9058, \\ 4. Ewa Tywanek, ewa.tywanek@gmail.com, https://orcid.org/0000-0002-2311-994X, \\ 5. Robert Jan Łuczyk https://orcid.org/0000-0003-0889-6326,
}

\section{Abstract}

It has been recently discovered that asprosin, an adopakine that is secreted by white adipose tissue, affects the release of glucose into the blood. When blood glucose levels rise, the production of asprosin is immediately suppressed. In healthy people, circulating asprosin shows daily fluctuations, and its concentration drops significantly with the beginning of eating. In patients with type 2 diabetes it was invastigated that both fasting and postprandial asprosin concentrations were significantly higher. Therefore, it is probable that elevated blood levels of asprosin and its impaired response to glucose levels fluctuation may be one of the causes of type 2 diabetes. It is considered, 
that administration of antibodies that block the action of asprosin may help diabetics reduce glucose levels. In addition, it can also contribute to the effective fight against obesity.

Key words: asprosin; adipokine; diabetes mellitus; obesity

\section{Introduction}

The International Diabetes Federation estimates that 425 million people worldwide suffer from diabetes and every 6 seconds a person dies due to its complications, making it the largest global epidemic of the 21 st century. The prediction is that the incidence of diabetes among adults may rise to 552 million by 2030 [1][2][3]. It is estimated, that more than $90 \%$ of diabetes cases are type 2 diabetes. So far, many clinical resaerches established that insulin resistance, pancreatic $\beta$-cell dysfunction and increased glucose secretion by the liver are involved in the pathogenesis of diabetes [2][3]. Therefore, in the treatment of diabetes it is important to use various drugs, the action of which counteracts the disease by various mechanisms corresponding to its pathogenesis.

Impaired insulin action in adipose tissue, liver or skeletal muscles, leads to insulin resistance, which is also linked to obesity [4]. Nevertheless, white adipose tissue has been recently recognized not only as an energy reservoir but also as an important secretory organ of adipokines [3][5]. Adipokines are hormones generelly considered to be involved in metabolic processes, such as inflammation, adipogenesis, angiogenesis, glucose homeostasis and insulin sensitivity. Some of them are also positively correlated with BMI and obesity-related biomarkers [4][6]. Due to the growing interest in adipokines and the increased amount of researches focused on white adipose tissue, a new type of adipokine was discovered - asprosin.

The concentration of asprosin in the blood fluctuates during the day and is dependent on glycemia - its level is elevated while fasting and it decreases quickly with the onset of eating. What distinguished asprosin from other adipokines, was its increased secretion and impaired response to glucose levels fluctuation in patients with insulin resistance and type 2 diabetes, regardless of eating [2]. It is still unknown what underlies this abnormality, but immunological and genetics factors were considered [2]. Taking into account the potential pathological role of asprosin in diabetes, it is speculated that administration of antibodies that block its activity may reduce glucose levels and contribute to the effective fight against obesity.

\section{Characteristics of asprosin}

Adipose tissue has recently attracted the attention of scientists for its ability to secrete various adipokines, which affect different metabolic processes. Several adipose tissue-secreted 
molecules can either enhance or impair insulin action [2]. For instance, resistin and leptin are known to antagonize insulin function in the liver and skeletal muscles, resulting in insulin resistance. In the contrary, adiponectin increases insulin sensitivity. Therefore, it appears that the balance between adipokines contributes to the maintenance of homeostasis [7]. In reference, the literature reports that mutations in some of the genes result in increased TGF- $\beta$ signaling, altered mechanical properties, impaired adipogenesis, leading to adipose tissue dysfunction and giving rise to many conditions, such as Marfan syndrome, marfanoid progeroid lipodystrophy syndrome and neonatal progeroid syndrome [8]. The need to explore this topic led to the discovery of a new adipokine.

Asprosin was identified in 2016 by Romere et al. during the genetic studies of patients with neonatal progeroid syndrome. It is a 140-amino-acid protein, produced in response to starvation by white adipocytes, as a C-terminal cleavage product of profibrillin (a pro-protein encoded by FBN1 gene) [3][7][9][10]. This process was confirmed by (i) RNA sequencing and qPCR data, demonstrating highly expressed FBN1 mRNA levels in white adipose tissue [8]. The factors that regulate its secretion are unknown [2][4].

The role of new adipokine is not fully understood, but so far it has been demonstrated that liver is its target organ, as asprosin modulates hepatic glucose release via the G protein-cAMPprotein kinase A signaling axis [2][7][8]. Single injection of recombinant asprosin leads to immediate increase of both blood glucose and insuline, but all study so far shows that asprosin does not directly affect insulin production in the pancreas [7][8]. Some reaserches suggested that exercise could also affect the levels of asprosin. Aerobic exercise training reduced the concentrations of asprosin in the liver of streptozotocin-induced diabetic rats. This consequently inhibited glucose production, TGF- $\beta$ levels and protein kinase A in the liver. However, this study was mainly limited to liver and the consequence of aerobic training on the skeletal muscles was not addressed. Moreover, another study reported that the single anaerobic effort induced an increase in asprosin secretion in women. These results indicate that the expression of asprosin in different tissue may be regulated by different factors and different pathways [8][9].

During the study of this new protein, its receptor in the liver was identified: it is known that by activating the olfactory receptor Olfr734, a G-protein coupled receptor of the rhodopsin family, asporin modulates hepatic glucose production. Surface plasmon resonance spectroscopy confirmed strong binding of asprosin to OLFR734 with a $\mathrm{K}_{\mathrm{D}}$ of $18 \mathrm{nM}$. This is in the range of circulating levels of asprosin under fasting conditions (5-15 nM). Moreover, it was observed that the Olfr734 deficiency significantly impaired both fasting and eating-induced glucose production and improved 
insulin sensitivity, what supports the idea of its critical role in maintaining glucose homeostasis [7] [8].

Except for the effect of asprosin on carbohydrate metabolism, it may also induce an inflammatory reaction. It was established that asprosin, depanding on dosage, is able to arouse tolllike receptor (TLR) 4 expression and JNK phosphorylation, enhacing the inflammatory response, cellular failure and promoting apoptosis in the pancreatic $\beta$-cells. As a result, insulin secretion of $\beta$ cells is reduced. What is more, asprosin impaired insulin sensitivity in skeletal muscle through inflammation pathways associated with $\mathrm{PKC} \delta[3][7][8][9]$.

Another reaserch showed that asprosin is capable of crossing the blood-brain barrier and exerting an orexigenic agouti-related peptide (AgRP) neurons in the arcuate nucleus of the hypothalamus via a cAMP-dependent pathway and simultaneously inhibiting anorexigenic POMC neurons, all of which mechanisms invoke stimulation of appetite, increase food intake and weight gain [2][8][9][10].

Taking into account the multidirectional action of asprosin, it can be expected that in the course of further research it will reveal other, hitherto unknown roles in maintaining homeostasis.

Fig. 1 [8]

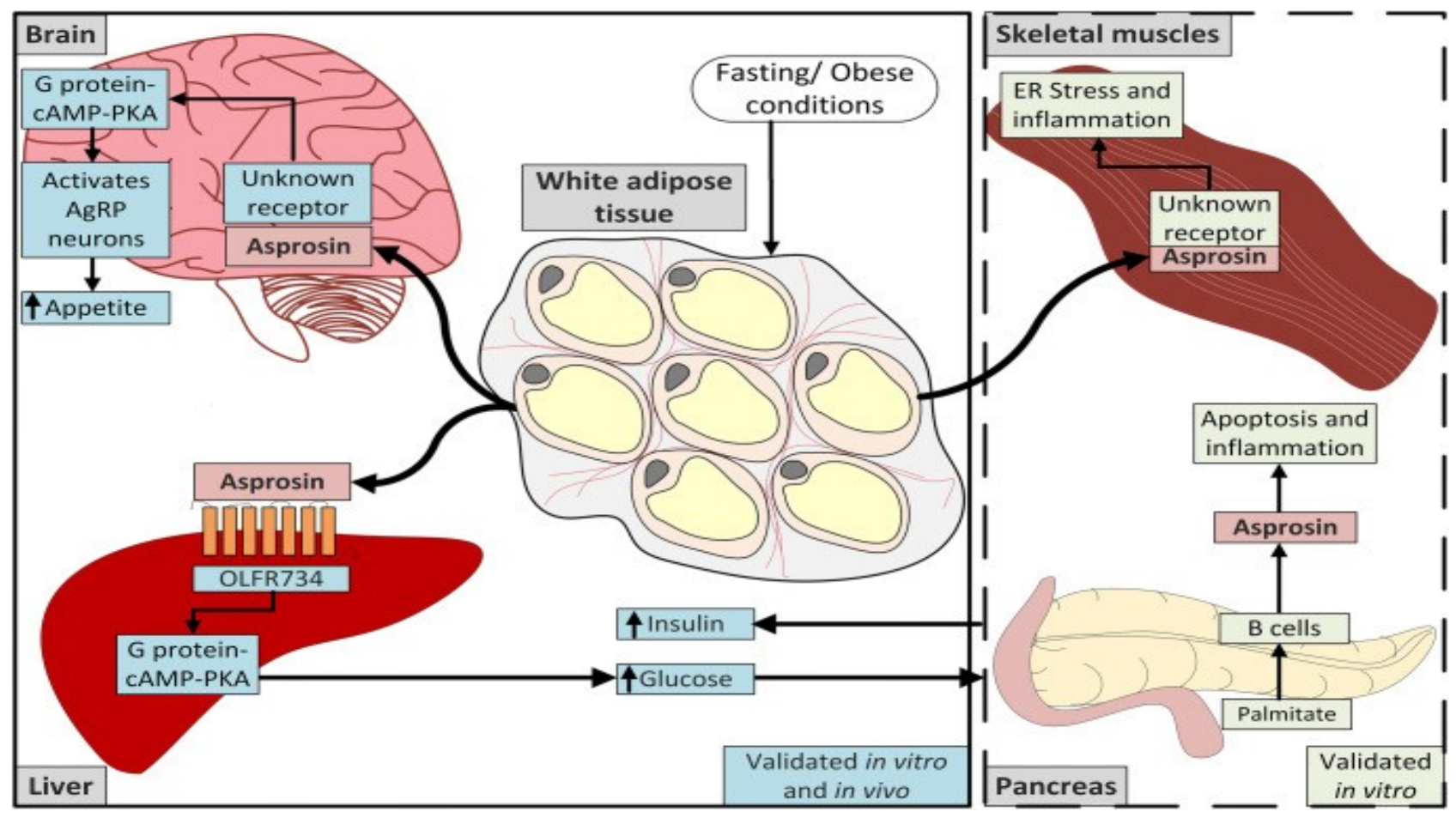

The role of asprosin in metabolic organs. Left solid box: During fasting or in obesity, increased circulating asprosin promotes hepatic glucose production and increases insulin secretion from the pancreas. Asprosin also activates the AgRP neurons in the brain and increases appetite. Right dashed box: Other in vitro studies have shown that increased asprosin levels lead to apoptosis and inflammation in pancreatic B cells and to inflammation and ER stress in the skeletal muscle cells [8]. 
Table $1[8]$

Summary of the principle roles of asprosin in different metabolic tissues and organs

\begin{tabular}{|c|c|c|}
\hline Tissue & Principle Function of Asprosin & References \\
\hline $\begin{array}{l}\text { Adipose } \\
\text { Tissue }\end{array}$ & Main source of asprosin production and secretion & Romere et al. 2016 \\
\hline Liver & Primary target tissue; promotes hepatic glucose production & $\begin{array}{l}\text { Romere et al. } 2016 \\
\text { Ko et al. } 2019\end{array}$ \\
\hline & Receptor binding (OLFR734) & Li et al. 2019 \\
\hline Pancreas & $\begin{array}{l}\text { Reduces insulin secretion; amplifies inflammatory response and apoptosis } \\
\text { of pancreatic cells }\end{array}$ & Lee et al. 2019 \\
\hline Brain & Increases appetite and food intake & $\begin{array}{l}\text { Duerrschmid et } \\
\text { al. } 2017\end{array}$ \\
\hline $\begin{array}{l}\text { Skeletal } \\
\text { Muscle }\end{array}$ & Augments endoplasmic reticulum stress and inflammation & Jung et al. 2019 \\
\hline
\end{tabular}

\section{Potential asprosin's role in the pathogenesis of diabetes}

It was speculated that the dysregulation of asprosin secretion by white adipose tissue and the impaired response of asprosin to glucose levels fluctuation might be one of the reasons for the onset of type 2 diabetes mellitus and the sustained high level of blood glucose in these patients [2][4][8].

So far, it has been proven, that asprosin is elevated in patients with insulin resistance, obesity and diabetes mellitus, but it is decreased in neonatal progeroid syndrome, characterized by a severe lipodystrophic phenotype [7]. In addition, in mice with streptozotocin-induced pancreatic $\beta$ cells demage, high blood glucose and lower asprosin levels were observed, compering to control group [8]. Therefore, it might be assumed that hepatic glucose production is increased due to the abnormal asprosin concentrations, which promotes the development of hyperinsulinemia and then worsens insulin resistance in the liver accordingly [2][4]. Study shows that plasma asprosin levels 
are significantly higher in subjects with impaired glucose regulation and type 2 diabetes mellitus compared with those with the normal glucose regulation group [3]. It is considered that aprosin, reaching the highest concetrations in group of patients with impaired glucose regulation, compering to those with normal glucose regulation as well as diabetes and acting unlike other adipokines, which rise linearly in all of these groups, indicates that this protein might be a strong biomarker to predict prediabetes [3].

Another important mechanism in the development of type 2 diabetes mellitus is most likely pancreatic $\beta$-cell malfunction and various adipokines have been already reported to affect the function, proliferation, apoptosis and failure of $\beta$ cell. Asprosin might also contributes to $\beta$-cell dysfunctin, inducing the glucose intolerance. Previous study demonstrated a direct effect of asprosin on hepatocyte glucose production without the potential insulin compensatory effect [3]. Additionally, study shows that asprosin is significantly higher in females with type 2 diabetes mellitus than in healthy subjects and is positively correlated with fasting blood glucose (FBG), glycated hemoglobin (HbA1c) and HOMA-IR [11], while in the controls, fasting glucose or HOMA-IR was not significantly associated with asprosin concentrations [4]. Another research found that serum asprosin concentrations were not sexually dimorphic, suggesting that it might not be affected by sex hormone status [4].

Some clinical trials have also investigated the relationship between asprosin and obesity, as it is a common comorbidity with type 2 diabetes. However, the results were not consistent [9]. Some of the reaseraches revealed that asprosin levels were significantly higher in obese adults as well as in children with obesity and insuline resistance. On the contrary, other scientists observed that plasma asprosin was significantly lower in obese children than in normal-weight children and there was a gender difference in its concentration [9].

However, it seems that both saliva and plasma asprosin concentrations are positively associated with adiposity-related parameters in patients with type 2 diabtes mellitus, including body mass index (BMI), waist circumference (WC) and waist-hip ratio (WHR). In the contrary, in nondiabetic subjects, no significant relationship was found between asprosin and these parameters [4] [7][8]. Following the increased asprosin concentrations, BMI, WC and WHR were gradually increased [4].

As it has been proven, excessive adiposity leads to dysfunction of adipokines and metabolic disorders, including dysfunction of $\beta$-cells [4][8]. Futhermore, in obesity, uncontrolled production of hepatic glucose is the main cause of hyperglycemia and ecessive hepatic glucose production depanding on asprosin could potentially lead to glucose toxicity, affecting pancreatic function. Therefore, reasearch suggest the role of asprosin in the deterioration of pancreatic function 
as a fundamental pathophysiological mechanism in type 2 diabetes mellitus [7]. Additionally, insulin resistance contributes to failure of pancreatic $\beta$-cells, resulting in decreased insulin secretion. Another problem asossiated with hyperglycemia is pancreatic inflammation that may be one of the causes for development of type 2 diabetes mellitus [8]. Also, in obese patients elevated circulating asprosin leads to increased appetite and food intake [8].

Data has shown a strong relationship between elevated plasma asprosin and lipids metabolism [4]. In combination with high hepatic glucose production in the insulin-resistant liver, dyslipidemia is a common medical condition. Given that the liver is the target organ of asprosin and insulin resistance can lead to dyslipidemia, it can be assumed that asprosin is involved in the pathogenesis of lipid disorders [4]. There are studies proving that asprosin is significantly correlated with serum triacylglycerol concentration and TC/HDL-C ratio (atherosclerotic risk factor of cardiovascular diseases) in type 2 diabetes patients [2][7][8]. However, there are no observations of a relationship between asprosin and HDL-C concentrations [7].

Diabetic kidney disease is one of the most severe complications of type 2 diabetes mellitus and has been the leading cause of the end-stage renal disease in the world. Hyperglycemia and insulin resistance are main causes of renal failure in these patients, though many other mechanisms have been involved in the pathogenesis of this condition, including ischemia, oxidative stress, inflammation, hemodynamic changes in kidneys and hyperfunction of renin-angiotensinaldosterone system [7]. Data suggest that asprosin is closely associated with the risk factors of diabetic kidney disease, such as hyperglycemia, insulin resistance and inflammation [9].

It has been shown, that anti-asprosin monoclonal antibodies reduce blood glucose, appetite, and body weight as well as improve insulin sensitivity, validating asprosin as a therapeutic target [4] $[10]$.

\section{Asprosin and other medical conditions}

Besides the relationship between asprosin and type 2 diabetes mellitus, the roles of asprosin in other diseases were also explored.

Patients with polycystic ovary syndrome, mainly obese women, often present with postprandial glucose abnormalities, which reflects peripheral insulin resistance and increases the risk of type 2 diabetes [11]. Moreover, sex-related hormones such as estradiol and SHBG are significantly correlated with asprosin, indicating that overweight / obesity leads to insulin resistance and then exacerbates the reproductive and metabolic features of PCOS. In addition, in all PCOS patients and in the overweight / obese PCOS subgroup, asprosine was positively associated with testosterone and prolactin [11]. However, similar to obesity-related markers, the results of 
relationship beetwen serum asprosin and PCOS were inconsistent. There are studies founding that circulating asprosin levels were elevated in women with PCOS and other ones that has not observed an abnormal serum asprosin levels in these patients [8][9]. Overall, most study shows that asprosin in PCOS subjects is often higher than in healthy subjects, but lower than in type 2 diabetes mellitus patients [11].

Some studies have found that higher asprosin concentrations before bariatric surgery were associated with a trend towards weight loss 6 months after surgery [2]. Futhermore, significatnly reduced plasma asprosin levels were related to weight loss after bariatric surgery, suggesting that asprosin could serve as an important marker in this surgical approach [8].

It has been observed, that in pregnant women with gestational diabetes or preeclampsia, the level of asprosin was significantly increased. Also, asprosin has been suggested to be a novel biochemical marker for predicting the severity of acute coronary syndrome with unstable angina pectoris, as its concentrations correlate with these states [2][8].

\section{Conclusions}

Taken together, most studies have proven that asprosin is elevated in patients with insulin resistance, obesity and diabetes mellitus and that anti-asprosin specific antibodies reduce blood glucose, appetite and body weight, that suggest that impaired function of asprosin may act as a trigger for new-onset type 2 diabetes. What seems to support this hypothesis is that plasma asprosin levels dynamically increases the blood glucose metabolism, correlating positively with fasting insulin and glucose concentrations, insulin resistance (HOMA-IR), HbA1c, markers of obesity and lipid profiles (LDL-c, TAG, TC, ApoB, ApoE), but negatively with $\beta$-cell function in type 2 diabetes mellitus patients [3][8][11]. It must be acknowledged that there are some differences in the assessment of the relationship between the concentration of asprosin and individual markers of metabolic disorders. However, ethnicity tends to influence adipokine levels and therefore results from studies conducted in different populations may lead to inconsistent conlusions. In order to be capable of concluding that the impaired response of circulating asprosin concentrations to glucose levels fluctuation is one of the causes of type 2 diabetes, futher reaserch should be carried out on larger populations.

\section{References:}

1. Yi-Ching W, Sung-Sheng T, Rong-Kuo L, Chun-Che Ch, Long-Sun R, Ming-Feng L, Hong-Shiu Ch, Chiung-Mei Ch, Jawl-Shan H, and Hung-Chou K, Diabetic Distal Symmetrical Polyneuropathy: Correlation of Clinical, Laboratory, and 
Electrophysiologic Studies in Patients with Type 2 Diabetes Mellitus, J Diabetes Res. 2020(8):1-11, originally published online $2020 \mathrm{Jul} 3$

2. Xinyue Z, Hui J, Xiaojing M, and Hongyan W, Increased serum level and impaired response to glucose fluctuation of asprosin is associated with type 2 diabetes mellitu, J Diabetes Investig. 2020 Mar; 11(2): 349-355. Published online 2019 Oct 3

3. Yuren W, Hua Q, Xin X, Yuyang Q, Yong L, Yingchun C, Yi Z, and Hongting Z, Plasma Asprosin Concentrations Are Increased in Individuals with Glucose Dysregulation and Correlated with Insulin Resistance and First-Phase Insulin Secretion, Mediators Inflamm. 2018:1-7. Published online 2018 Mar 20

4. Lei Z, Chao C, Nan Z, Yuming F, Xingbo C, Circulating asprosin concentrations are increased in type 2 diabetes mellitus and independently associated with fasting glucose and triglyceride, Clinica Chimica Acta 2017(489):183-188

5. Azamar-Llamas D, Hernández-Molina G, Ramos-Ávalos B, Furuzawa-Carballeda J, Adipokine Contribution to the Pathogenesis of Osteoarthritis, Mediators Inflamm. 2017(5468023):26, Published online 09 Apr 2017

6. Helfer G, Qing-Feng W, Chemerin: a multifaceted adipokine involved in metabolic disorders, , J Endocrinol 2018 Aug;238(2):R79-R94

7. Shakiba N, Mohsen N, Mehdi Z, Moslem L N, Ali G, Mohammad R B, Hafez $\mathrm{H}$, and Mohammad M, Serum concentration of asprosin in new-onset type 2 diabetes, Diabetol Metab Syndr. (2020) 12:65. Published online 2020 Jul 23

8. Muthu L M, Dieter P R, Fibrillin-1 and fibrillin-1-derived asprosin in adipose tissue function and metabolic disorders, J Cell Commun Signal 2020 Jun;14(2):159-173

9. Hongyu Z, Wenqi H, Guang Z, Circulating asprosin levels are increased in patients with type 2 diabetes and associated with early-stage diabetic kidney disease, International Urology and Nephrology 2020, 52:1517-1522

10. Hoffmann J G, Xie W, Atul R C, Energy Regulation Mechanism and Therapeutic Potential of Asprosin, Diabetes 2020 Apr;69(4):559-566

11. Xing L, Mingyu L, Rufei S, Linlin Z, Hua H, Jun W, Xiuli W, Hua Q, Shaodong G, Min L and Hongting Z, Plasma Asprosin Levels Are Associated with Glucose Metabolism, Lipid, and Sex Hormone Profiles in Females with Metabolic-Related Diseases, Mediators Inflamm. 2018(1):1-12. Published online 2018 Nov 6 